\title{
Teoría y Práctica del Ordenamiento y Manejo Sustentable del Territorio: Tijuana-Rosarito-Tecate, Baja California, México
}

Francisco R. Venegas y Rosa I. Rojas

Universidad Autónoma de Baja California, Facultad de Arquitectura, Bulevar Benito Juárez y

Calle de la Normal s/n Mexicali, B.C.-México (rvenegas@uabc.mx, rosa_rojas@uabc.mx)

\begin{abstract}
Resumen
El objetivo de este trabajo, fue la aplicación del ordenamiento territorial y el manejo sustentable de los recursos bajo la perspectiva del modelo ciudad-región. El ordenamiento territorial permite conciliar los intereses de los diferentes sectores de la economía y que junto con el desarrollo sustentable permiten garantizar un desarrollo equitativo entre las diversas actividades que soportan la economía y la protección de medio ambiente. La aplicación del ordenamiento territorial y los sistemas de información geográfica permitieron definir la aptitud territorial, identificar potencialidades para el aprovechamiento y conservación de los recursos en el espacio urbano-regional, y proponer esquemas para el manejo sustentable en el modelo ciudad-región Las conclusiones del estudio plantean limitaciones y oportunidades que enfrenta este modelo en la práctica de la planeación urbana.
\end{abstract}

\section{Theory and Practice of the Spatial Planning and Sustainable Territorial Management: Tijuana-Rosarito- Tecate, Baja California, México}

\begin{abstract}
The objective of this work was the application of the spatial planning and sustainable management of resources under the perspective of the city-region model. Spatial planning allows to balance the interests of the different sectors of the economy and in conjunction with sustainable development allow guarantying an equitable development between the diverse activities that support the regional economy and the required environmental protection. Application of spatial planning and geography information systems allowed defining the territorial characteristics, identifying potentialities for the better use and preservation of the resources in the urban-regional space. The main conclusions of the study are related to the limitations and opportunities that this model faces in practice.
\end{abstract}

Keywords: spatial planning, sustainable management, city-region model, urban-regional planning 


\section{INTRODUCCIÓN}

El noroeste de Baja California (B.C.) México, con un ecosistema mediterráneo, único en el país, es la región con mayor crecimiento y desarrollo en el estado, y que en las últimas cuatro décadas, casi triplicó su población ya que en 1970 habitaban en el estado, 870421 habitantes, al año 2000 era de 2.48 millones y al 2005 fue de 2.84 millones, con tasas de crecimiento de 5.5, 4.15 y 2.4 respectivamente (CONEPO, 2003; INEGI, 2006a; Ojeda-Revah et al., 2008).

Este incremento poblacional se dio por un impulso del Gobierno Federal hacia un proceso de industrialización de la frontera norte, por ejemplo en 1985 en B.C., se tenían registradas 307 maquiladoras, en 1996 se incrementó a 793, la más alta del país, pasando a ocupar el primer lugar de este tipo de empresas a nivel nacional con un 33\% del total (INEGI, 1999b; INE-SEMARNAP, DGE, 2000).

El otro gran factor que ha promovido ese gran incremento población, ha sido la masiva migración que desde las décadas de los setentas y ochentas ha experimentado toda la frontera norte del México (Binational Study, 1997; Bustamante, 2000; Coubès, 2003), asimismo esta región mantiene una fuerte y estrecha interacción económica, social y comercial con la ciudad de San Diego, California en los Estados Unidos de Norteamérica (McGeough et al., 2004)

Este crecimiento poblacional ha promovido la conurbación de las ciudades de Tijuana, Playa de Rosarito y Tecate (ZMTPRT), conformando la quinta zona metropolitana en el país (SEDESOL, 2001) y debido a la rapidez con que se han presentado estos cambios, se han empezado a manifestar problemáticas comunes en los tres municipios relativas a la desigualdad en la infraestructura instalada y del equipamiento urbano, así como en el tratamiento y manejo de los desechos sólidos municipales, deficiente articulación de vialidades y transporte, marginación y deterioro ambiental.

Además, esta conformación del espacio urbano, está enmarcada por procesos económicos de globalización, tendencias políticas de descentralización, así como una intensa movilidad poblacional (Castaños, 2005). El resultado, expansión periférica, proliferación de asentamientos aislados, con la consecuente absorción y degradación ambiental y agotamiento de los recursos naturales en el área de influencia de las grandes concentraciones urbanas.

Estos problemas han propiciado la necesidad de contar con un modelo de gestión del área metropolitana que permita en forma conjunta a los administradores de las diversas dependencias de gobierno federal, estatal y a otros agentes del desarrollo urbano, proponer y realizar proyectos de planeación y gestión del territorio de esta zona metropolitana.

Este no es problema únicamente de México, ya que en la mayoría de los países en desarrollo es un problema común, al grado que la mayoría de los países latinoamericanos y europeos han propuesto la aplicación del ordenamiento territorial, como la herramienta de planeación ambiental que pretende en lo posible armonizar la oferta ambiental del territorio (Rosete, 2006; United Nations, 2008) y evitar conflictos entre los diferentes usuarios bajo el paradigma del desarrollo sustentable.

Esta preocupación surgió a partir de la primera reunión cumbre de Estocolmo en 1972, también conocida como la Conferencia de Estocolmo, en la cual se empiezan a encender focos rojos sobre el deterioro ambiental, el avance de la desertificación, el incremento de la contaminación y el crecimiento exponencial de la población humana.

A partir de esta primera reunión cumbre en Estocolmo de la UNEP (United Nations Environmental Program), México como país miembro se compromete a poner en práctica políticas que ayuden a detener y revertir el deterioro ambiental en su territorio. Para cumplir con estos compromisos en 1988 publicó un documento que se llamó; Manual de Ordenamiento Ecológico del Territorio. Posteriormente en el año 2000 se publica otro documento llamado; Términos de Referencia Generales para la Elaboración del Programa Estatal de Ordenamiento Territorial (SEMARNAP. SEDESOL-CONEPO-INEGI, 2000). 
Rosete (2006); Venegas y Rojas (2006) y el INE-SEMARNAT (2006) presentan una revisión del origen y evolución que ha tenido el ordenamiento territorial en México y Latinoamérica y el uso de los sistemas de información geográfica en la ordenación del territorio y la definición de la aptitud territorial.

Bajo esta perspectiva, el objetivo principal de esta investigación fue la obtención de la aptitud territorial a través de la aplicación del ordenamiento territorial (OT) y el manejo sustentable de los recursos utilizando el modelo ciudad-región.

\section{MATERIALES Y MÉTODOS}

Para alcanzar los objetivos propuestos se planteo una estrategia metodológica, la cual se base en tres enfoques estrechamente relacionados con la sustentabilidad ambiental.

El primer enfoque se basa en el ordenamiento y regulación del territorio a partir de las potencialidades y restricciones físicas y biológicas para soportar diferente tipo de actividades de desarrollo y conservación. Esta primera fase se hizo a través de la aplicación de la metodología del Manual de Ordenamiento Ecológico del Territorio (SEMARNAT, 2002), que es un instrumento de política ambiental mexicana, que por ley se debe de aplicar en todo el territorio nacional para la definición de los usos del suelo.

Este primer enfoque, se hizo a través del método geopedológico de Zinck (1988), conjuntamente con la utilización de métodos sintéticos (Rossiter, 2000). La aplicación de ambos métodos permitió definir las unidades de paisaje, que son la síntesis de una serie de variables del medio ambiente y procesos exógenos o geomorfológicos.

Para la obtención de la aptitud territorial se partió del análisis de la geología superficial de Gastil et al. (1975), y el tectonísmo (O'Connor y Chase, 1989; Kier et al., 2001; Cruz-Castillo, 2002). Este análisis se basa en la separación de formaciones geológicas que por su origen se les clasifico como estables, es decir materiales de origen intrusivo, como los granitos y los de origen extrusivo, producto de actividad volcánica. Lo mismo se hizo con los materiales denominados como inestables, producto de intensos procesos de erosión que se presentaron desde el Cretácico, continuaron durante todo el Terciario y que a la fecha se siguen manifestando, dando origen a Formaciones de conglomerados o materiales de depósito, mal clasificados y fácilmente erosionables.

Una vez hecha la separación de materiales estables e inestables, se procedió a obtener las pendientes de los modelos digitales de elevación (MDE) (INEGI, 1999a). Estos MDE, se manipularon para obtener un archivo de pendientes a través de un Sistemas de Información Geográfica (SIG) IDRISI (Eastman, 2003). De los MDE se obtuvieron tres rangos de pendientes en porcentaje, de la siguiente forma $0-15 ; 15-35$ y 35 al máximo valor esperado en toda la región.

Todos los rangos de pendientes se cruzaron con cada uno de los materiales estables e inestables, este cruce permitió separar e identificar, cuales son las zonas urbanas y no urbanas que presentan desde riesgo alto por su inestabilidad, hasta las que son altamente estables inclusive con rangos de pendientes arriba de $35 \%$.

Otra fuente de información que se utilizó para identificar las zonas susceptibles a sufrir deslizamientos, corresponde a las zonas que son cruzadas por fallas y arroyos. A cada una de estas estructuras se le generó un buffer de 250 metros a ambos lados a través de todo su trazó visible.

La manipulación de la toda la información a la que se ha hecho referencia permitió obtener la aptitud territorial obtenida. La cual se considera el insumo básico para la ordenación del territorio.

El segundo enfoque, se basa en el modelo de Indicadores Ambientales desarrollado por la Organización Económica para la Cooperación y el Desarrollo (OECD, 1993) la cual plantea el 
diagnóstico ambiental a través del uso de indicadores de sustentabilidad, basado en el modelo de Presión-Estado-Respuesta (PER).

Para alcanzar esta meta se partió de la elaboración del diagnóstico ambiental, basado en el manejo de indicadores de sustentabilidad urbana, a través de considerar el manejo de los recursos naturales que soportan las actividades económicas y sociales, como parte del metabolismo urbano, es decir las ciudades demandan una serie de insumos como el agua, energía y alimentos, insumos que son indispensables para su sostenimiento. Estos insumos una vez utilizados generan desechos que salen de la ciudad como contaminantes de la atmósfera, del suelo, del agua y que influyen en la pérdida de biodiversidad. La estrategia para evitar en lo posible riesgos con la producción de estos desechos, es la de manejarse adecuadamente a través de reducirlos, reutilizarlos, reciclarlos y rehabilitarlos para tener una mejor eficiencia en el manejo del agua residual, drenaje, calidad del aire, residuos sólidos municipales, áreas productivas, áreas verdes y de conservación y minimizar posibles riesgos naturales e inducidos.

El tercero enfoque, es una estrategia de ordenamiento territorial por niveles jerárquicos que van desde ámbito doméstico, el regional y nacional. Se basa en el manejo sustentable de los recursos a partir de los modelos de ciclo cerrado de Tjallingii (1995), de la ciudad sustentable que promueve la reducción del consumo, la reutilización de recursos y reciclado de los mismos, desde diferentes escalas territoriales para el manejo de los flujos de agua-drenaje, energía, y residuos sólidos, en tanto que para los modelos de las áreas verdes y de conservación y de tráfico-transporte, se desarrollaron modelos basado en la estructura jerárquica propuesta por el mismo autor.

\section{RESULTADOS}

\section{Localización geográfica y factores ambientales}

La zona metropolitana de Tijuana-Playas de Rosarito-Tecate, se localiza en el noroeste de Baja California, México y sus coordenadas en UTM Zona 11, son: 486688 y 558995 Este y 3549179 y 3607304 Norte. (Fig. 1) La superficie total comprende las tres cabeceras municipales Tijuana, Rosarito y Tecate que suma un total de 117,884 has, equivalente al $1.68 \%$ del total de la superficie del estado y concentra el $55 \%$ de la población total de la entidad.

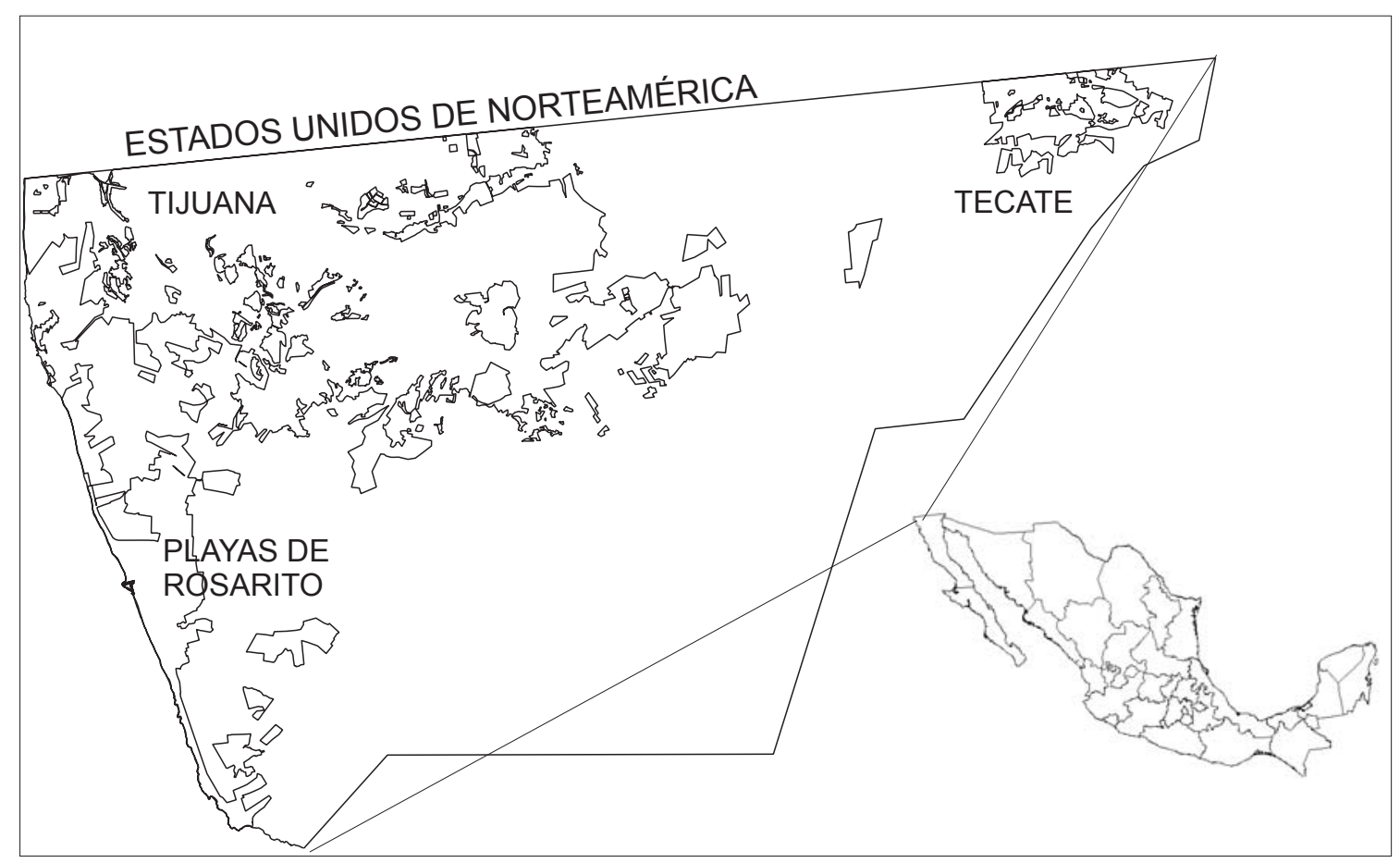

Fig. 1: Localización área de estudio Fuente: SEDESOL-SIDUE-COLEF (2003). 
La ZMTPRT Pertenece a la provincia fisiográfica de la península de Baja California, sub-provincia Sierras de Baja California Norte, caracterizada por elevaciones que van del nivel del mar, hasta los $1,280 \mathrm{msnm}$. La zona pertenece al batolito peninsular de edad Mesozoica, con rocas ígneas extrusivas e intrusivas de origen volcánico; presenta también materiales de edad Terciario, es decir areniscas y conglomerados, producto de un intenso proceso de erosión que inicio desde el Cretácico (Gastil et al., 1975), como resultado del tectonísmo.

El área de estudio se localiza en una de las tres regiones con mayor riesgo sísmico del estado, las principales fallas geológicas son; Vallecitos, García, Agua Caliente y la Nación, que cruzan la ciudad de Tijuana, sobre el cauce del río Tijuana y la Presa Abelardo L. Rodríguez y la Mesa de Otay (CruzCastillo, 2002). El clima de la región es de tipo mediterráneo BS, caracterizado por inviernos fríos y lluviosos, veranos secos y calientes (García, 1981), temperatura media anual van de 16.8 a $17.5^{\circ} \mathrm{C}$ y 220 a $270 \mathrm{~mm}$ de precipitación. Bajo estas condiciones climáticas se puede distinguir vegetación de zonas costeras y vegetación de marismas y dunas. Mientras que hacia el interior se establece el matorral costero, chaparral y bosque de coníferas; este último localizado principalmente en las altas montañas. En la actualidad el cambio en el uso del suelo ha sido responsable del impacto negativo que ha tenido la vegetación, con la consecuente reducción de la superficie de la vegetación nativa (INE-SEMARNAP-I.G.-UNAM, 1999).

\section{Ordenación de la Zona Metropolitana Tijuana, Playas de Rosarito-Tecate}

En este trabajo se presentan los resultados sintetizados del documento que se elaboró para la SIDUE-SEDESOL-UABC/ISS (2005), sobre la Sustentabilidad de la ZMTPRT, para hacer propuestas de uso del suelo sobre la base de la sustentabilidad regional y urbana, y el modelo ciudad-región, ya que no se puede concebir un crecimiento y desarrollo ordenado y sustentable si no están implícitos los flujos de energía en ambas direcciones.

En los resultados de la primera fase se presenta la aptitud territorial que es el insumo básico para los planes de ordenamiento del territorio. La aptitud territorial se obtuvo de la separación de las diferentes formaciones geológicas, sus edades y los paisajes, así como la presencia del levantamiento tectónico y que conjuntamente con eventos hidrometeorológicos promovieron intensos procesos de erosión, que iniciaron desde el Cretácico Superior y que en la actualidad se siguen manifestando (Gastil et al., 1975).

El resultado, unidades cartográficas que muestran la distribución de las Formación de conglomerados no consolidados e inestables, que son materiales arenosos de texturas gruesas y medias, fácilmente erosionables, que han provocado intensos movimientos de masa o deslizamientos, destruyendo infraestructura instalada, equipamiento urbano, así como la pérdida de vidas humanas y sus propiedades, debido a que gran parte de la zona urbana de la ZMTPRT, esta asentada sobre este tipo de materiales, siendo uno de los principales problemas ambientales que se presenta con mayor recurrencia en la ciudad de Tijuana, sobre todo en la época de lluvias. (Fig. 2)

A las unidades cartográficas obtenidas de la separación de los materiales estables e inestables o conglomerados, se cruzaron con archivos de pendientes separadas en cinco rangos, desde las zonas planas hasta las de topografía muy accidentada. Esta última información se obtuvo de la manipulación de los modelos digitales de elevación (INEGI, 1999a).

A continuación, a través de la utilización de los SIGs, por medio de la sobreposición cartográfica y mediante la funciona booleana AND, se integraron las fallas activas para separar zonas por tipos de materiales, estables e inestables, por rangos de pendientes y por la presencia o ausencia de fallas activas, dando como resultado diferentes rangos de riesgo, desde muy alto, no adecuadas para desarrollo urbano, hasta las áreas sin riesgo y aptas para este tipo de uso del suelo. Asimismo se sobrepusieron con la función AND, las zonas susceptibles de recarga de acuíferos y de las comunidades vegetales del Inventario Nacional Forestal (INE-SEMARNAP-I.G.-UNAM, 1999), lo que permitió definir las zonas que deberán tener un estatus de uso productivo a través de agricultura de riego y de conservación de comunidades vegetales que por su extensión están a punto de ser eliminadas. El resultado, la aptitud territorial de la ZMTPRT. (Fig. 3) 


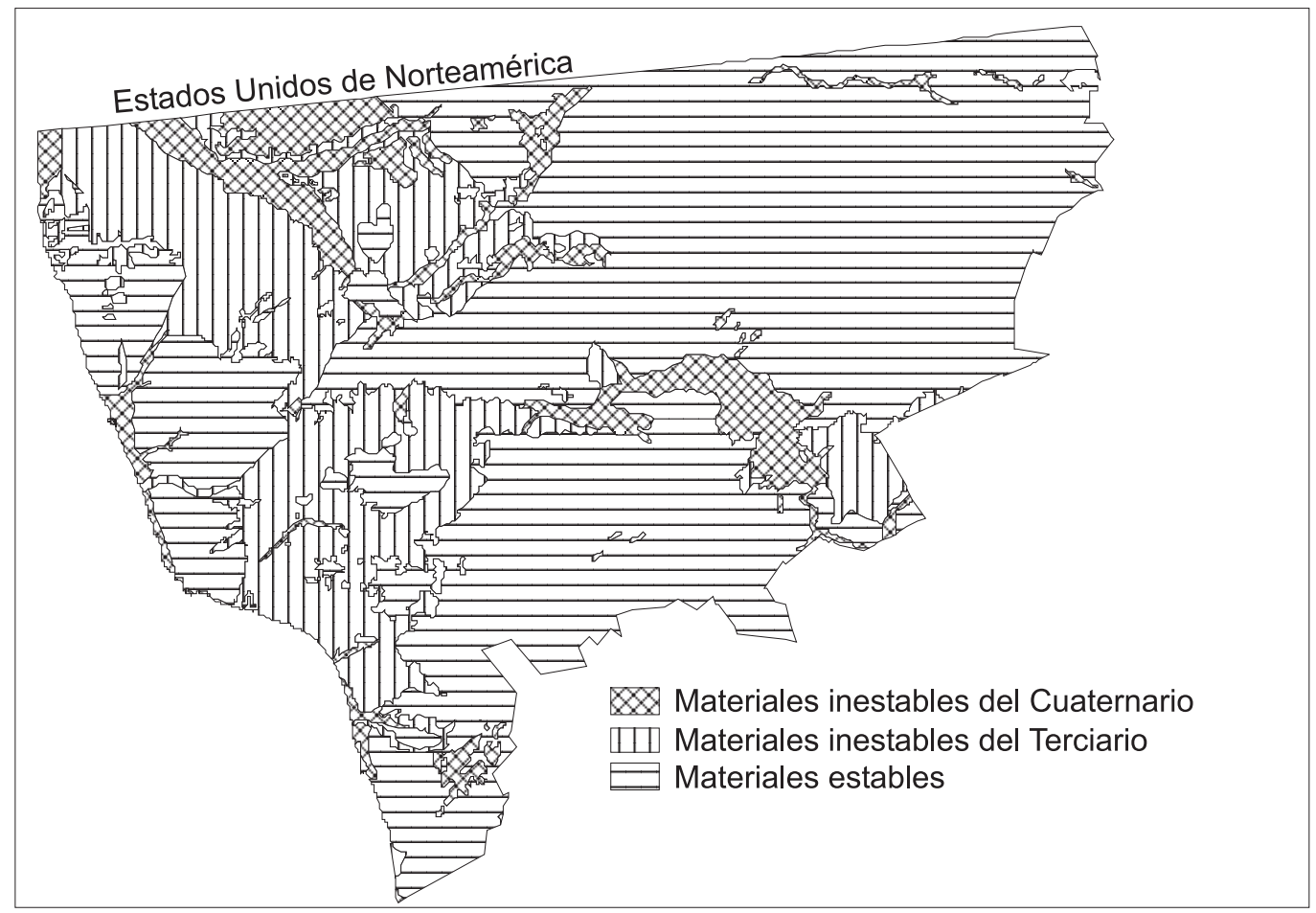

Fig. 2: Materiales estables e inestables (Fuente Gastil et al., 1975)

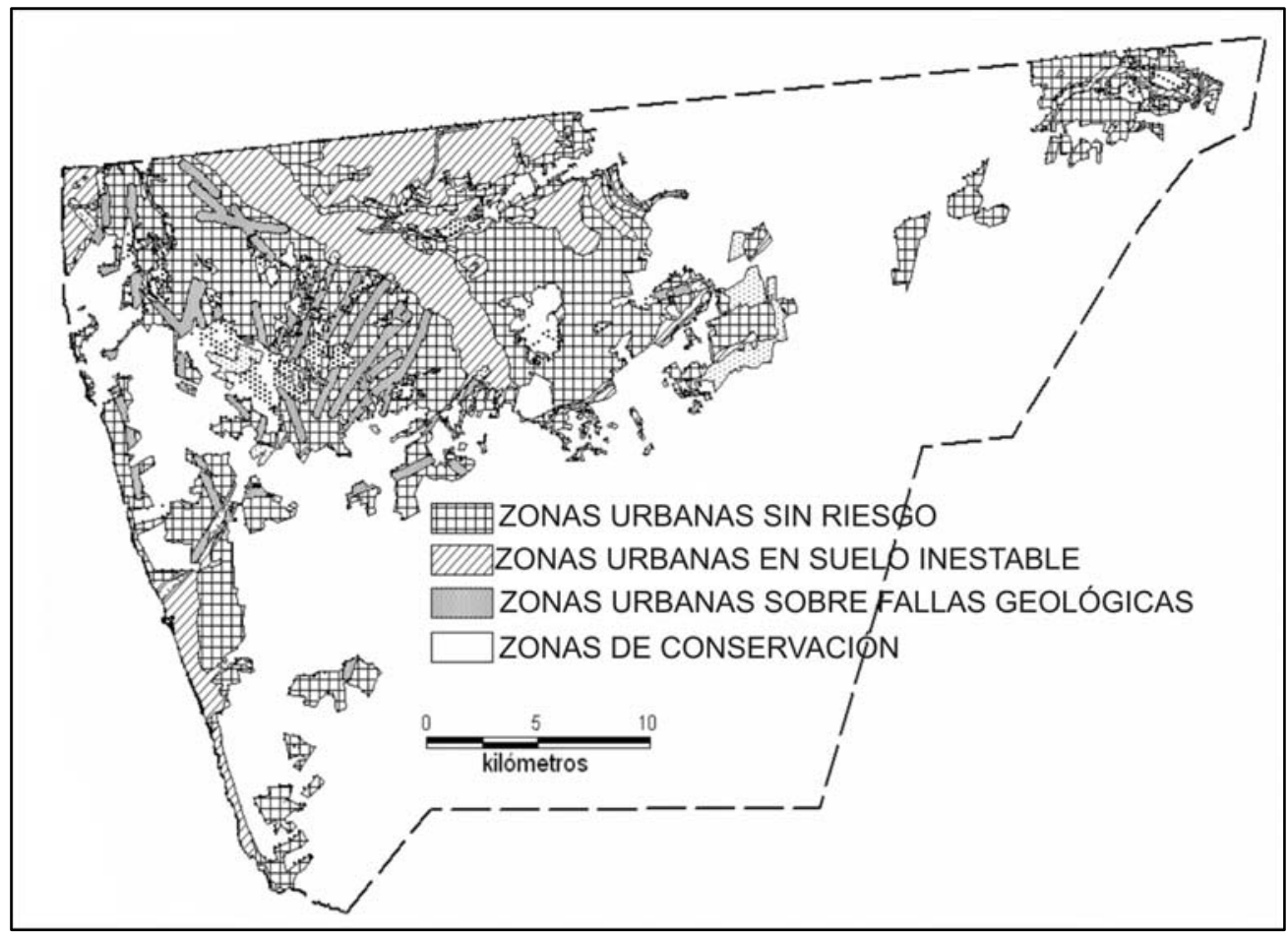

Fig. 3: Aptitud territorial

Diagnóstico ambiental: indicadores de sustentabilidad

Para la caracterización de la ZMTPRT en materia de sustentabilidad ambiental se utilizó el modelo de Indicadores Ambientales desarrollado por la OECD (1993), conocido como Presión-EstadoRespuesta (PER). El modelo PER ofrece un marco a través del cual se plantean las causas y los efectos que tienen las actividades productivas y de asentamientos humanos sobre el ambiente y como la sociedad reacciona ante ello para responder con acciones que contribuyan con su prevención, reducción, recuperación, mejoramiento y regulación. El modelo consta de tres grupos de indicadores: 
(P) Se refiere a la presión directa e indirecta que ejercen las actividades humanas sobre el ambiente, así como el funcionamiento propio de los sistemas naturales por ejemplo; incremento de población, políticas sectoriales, cambios tecnológicos, aprovechamiento de recursos naturales, agricultura, industria, transporte, ciclos de nutrientes y eventos naturales como el fenómeno de El Niño que se traduce a intensas lluvias o La Niña, periodos recurrentes de sequía por 4 años, así como la probabilidad de actividad sísmica y la presencia de masas de aires húmedo y frió que se traducen en lluvias intensas provocando deslizamientos de terreno.

(E) Este indicador se traduce al estado que presenta el ambiente, una vez que las presiones han incidido y han casado alteración o modificación en los ecosistemas y como estos cambios repercuten en la población.

(R) Se concreta a las acciones de las políticas, los instrumentos y la participación que la sociedad genera para contrarrestar las presiones y efectos sobre el ambiente, producto del desarrollo. Las respuestas se elaboran y se llevan a la práctica por los sectores gubernamentales, privados y sociales dentro de un marco legal nacional o internacional.

Además de lo anterior se pudieran agregar algunos aspectos de la gestión, relativos a la capacidad y desempeño para la aplicación de normas y ejecución de planes y proyectos con énfasis en la sustentabilidad (Tabla 1, Fuente: Modificado a partir de indicadores urbanos y sustentables de los autores: Seattle/King County (1998); Leitmann (1994) y Sanz (2000).)

Tabla 1: Diagnóstico a través del manejo de indicadores: residuos sólidos

\begin{tabular}{|c|c|c|c|}
\hline \multirow{13}{*}{ 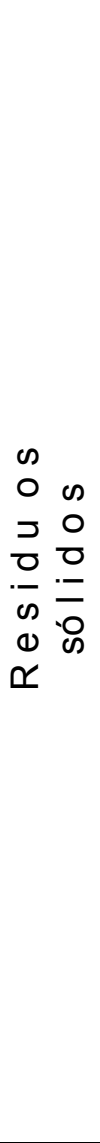 } & Presión & Estado & Respuesta \\
\hline & $\begin{array}{l}\text { Generación de residuos } \\
\text { municipales per cápita }\end{array}$ & $\begin{array}{l}\text { Cobertura del servicio de } \\
\text { recolección }\end{array}$ & $\begin{array}{l}\text { Recuperación ambiental de } \\
\text { sitios de disposición final }\end{array}$ \\
\hline & $\begin{array}{l}\text { Volumen de generación de } \\
\text { residuos no peligrosos }\end{array}$ & $\begin{array}{l}\text { Capacidad de los sitios de } \\
\text { disposición final }\end{array}$ & $\begin{array}{l}\text { - Programa para el manejo } \\
\text { integral de residuos sólidos }\end{array}$ \\
\hline & $\begin{array}{l}\text { Volumen de generación de } \\
\text { residuos peligrosos }\end{array}$ & $\begin{array}{l}\text { Capacidad de las } \\
\text { estaciones de transferencia }\end{array}$ & Reglamentación local \\
\hline & $\begin{array}{l}\text { Volumen de generación de } \\
\text { escombro }\end{array}$ & $\begin{array}{l}\text { Otras formas de disposición } \\
\text { de los residuos }\end{array}$ & Normas oficiales \\
\hline & $\begin{array}{l}\text { Vol. de generación de } \\
\text { residuos biológico infecciosos }\end{array}$ & Basureros a cielo abierto & $\begin{array}{l}\text { Programas especiales de } \\
\text { recolección }\end{array}$ \\
\hline & & $\begin{array}{l}\text { Población expuesta a la } \\
\text { contaminación }\end{array}$ & $\begin{array}{l}\text { Incentivos económicos a } \\
\text { empresas recicladoras }\end{array}$ \\
\hline & & Superficies contaminadas & \\
\hline & & $\begin{array}{l}\text { Porcentaje de residuos } \\
\text { reciclados }\end{array}$ & \\
\hline & & Empresas recicladoras & \\
\hline & $\begin{array}{l}\text { Consumo de materiales de } \\
\text { construcción per cápita }\end{array}$ & $\begin{array}{l}\text { Número y tipo de bancos de } \\
\text { materiales }\end{array}$ & Marco jurídico ambiental \\
\hline & $\begin{array}{l}\text { Extracción de materiales para } \\
\text { la construcción }\end{array}$ & $\begin{array}{l}\text { Superficies de bancos de } \\
\text { materiales abandonados }\end{array}$ & $\begin{array}{l}\text { Evaluación de Impacto } \\
\text { Ambiental de proyectos }\end{array}$ \\
\hline & & $\begin{array}{l}\text { Industrias de uso intensivo de } \\
\text { recursos naturales }\end{array}$ & Normas oficiales \\
\hline
\end{tabular}

\section{Esquemas para el manejo sustentable de recursos}

El tercer enfoque, se basa en los modelos de ciudad sustentable de Tjallingii (1995). Partiendo del enfoque de este autor, se tiene que las ciudades contienen organismos que corresponden a poblaciones humanas, especies animales y vegetales, entre los cuales existen relaciones complejas, que se denominan ecosistemas urbanos y bajo este nivel de relaciones entre los diferentes organismos y el medio que habitan, se establecen flujos de energía que se utilizan para su 
sostenimiento y sus diferentes áreas de influencia, es decir entre la ciudad y las zonas periféricas o rurales que la rodean.

En este trabajo se presentan los modelos: agua-drenaje y del sistema de áreas verdes, productivas y de conservación que muestran como podrían instrumentarse otros modelos de ciclo para el manejo sustentable de recursos en la ciudad-región (Tjallingii, 1995).

\section{Modelo de agua-drenaje}

Este modelo presenta en el nivel de edificación, la reducción del consumo de agua potable para los diferentes usos en la vivienda mediante el uso de accesorios reductores, en grifos, sanitarios, lavadoras, regaderas. Asimismo, plantea el uso de tuberías separadas para el desalojo de aguas grises, negras y pluviales, que permiten conducir las aguas para su reutilización doméstico o bien mandarlas fuera de la ciudad y conducirlas hacia plantas de tratamiento diferenciado. El procedimiento se repite y articula con el nivel de barrio o distrito, donde se captan los drenajes pluviales de calles y se mandan a cuerpos de agua existentes o para reutilización en el riego de áreas verdes públicas, en tanto que las aguas negras se van a un separador de lodos.

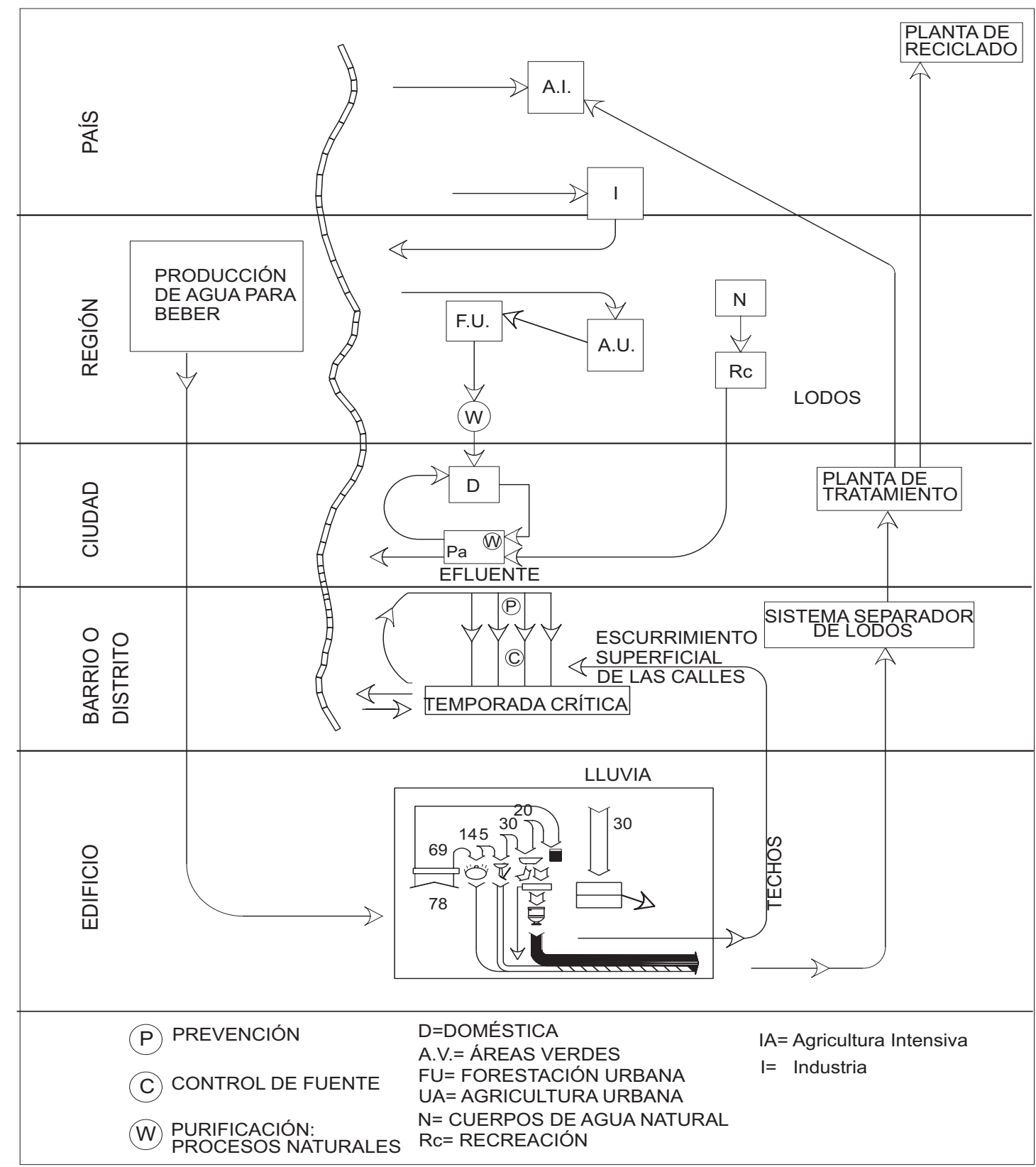

Fig. 4: Modelo Agua-drenaje Fuente: Modificado a partir de Tjallingii (1995) 
Posteriormente, las aguas negras se van a la planta de tratamiento de la ciudad y una vez que han sido tratadas, se pueden utilizar en diferentes actividades urbanas: recarga de acuíferos, forestación, áreas verdes, recreación, agricultura urbana, industria, cuerpos de agua. Mientras que los residuos sólidos como lodos, se pueden incorporar a las actividades de agricultura intensiva en otras regiones (Fig. 4).

\section{Modelo sistema de áreas verdes, productivas y de conservación}

Este esquema, al igual que el anterior, muestra una jerarquía en el sistema de áreas verdes, productivas y de conservación que ligados a las estructuras viales, canales y cuerpos de agua se puede articular para su manejo desde el nivel doméstico hasta el regional y nacional. En todas ellas se debe de promover la forestación con fines ambientales y estéticos. Así se pueden apreciar en la figura los diferentes valores y funciones que tienen las áreas verdes en la ciudad-región y la necesidad de su manejo integrado y coordinado por los diferentes niveles de gobierno y sociedad (Fig. 5).

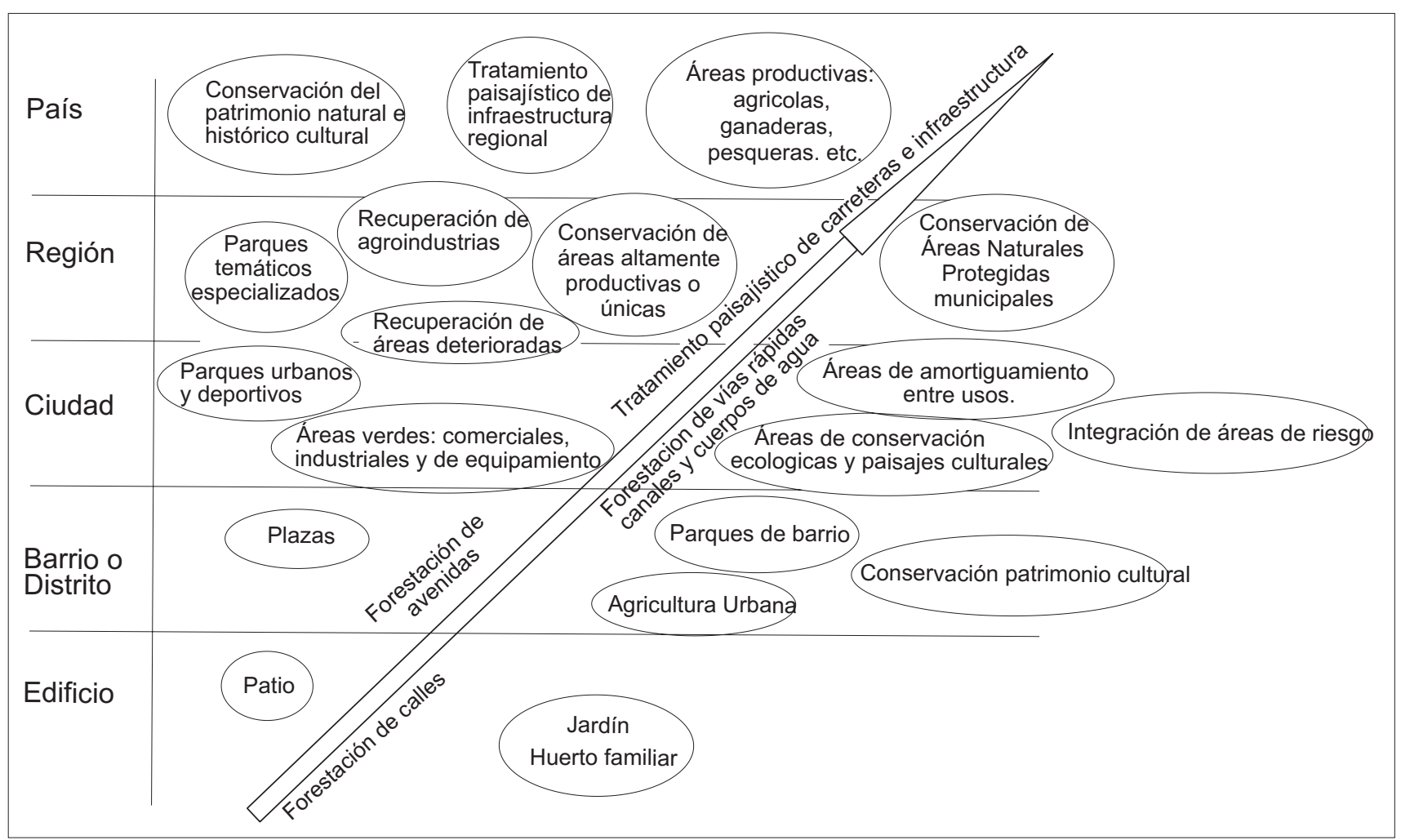

Fig. 5: Modelo sistema de áreas verdes, productivas y de conservación Fuente: Modificado a partir de Tjallingii (1995)

\section{DISCUSIÓN}

En esta sección se analizan y se discuten las diferentes posiciones, enfoques y resultados que se tienen en la elaboración de planes y programas de ordenamiento de los usos del suelo en el estado de Baja California. Para ello se revisaron y contrastaron; el Plan de ordenamiento ecológico del Estado de Baja California (DGE., 1995) y el Programa Estatal de Desarrollo Urbano y Ordenamiento territorial (SEDESOL-SIDUE-COLEF, 2003)

Estos dos instrumentos presentan una serie de inconsistencias generadas en primera instancia por las fuentes consultadas, debido a que en la elaboración de ambos documentos se utilizó la cartografía temática del Instituto Nacional de Estadística Geografía e Informática (INEGI, 2001), organismo público encargado de generar, la información estadística, y geográfica, así como la cartografía oficial de toda la República Mexicana, en escalas 1:50,000 y 1:250,000, principalmente.

La mayor parte de esta cartografía temática de todo el país se generó a partir de fotografía aérea 
escala 1:70,000 de 1973 y fue publicada en 1982 en escalas 1:250,000, por lo que en la actualidad la carta temática de Usos del suelo y vegetación resulta totalmente obsoleta, y en especial para la zona fronteriza del estado de Baja California, el uso del suelo ha cambiado drásticamente.

La nula aplicación de criterios científicos basados en procesos geomorfológicos en el Plan de Ordenamiento Ecológico del Estado de Baja California (DGE, 1995) y Programa Estatal de Desarrollo Urbano y Ordenamiento territorial (SEDESOL-SIDUE-COLEF, 2003), han contribuido para una reconversión de los usos del suelo con la desaparición de valles agrícolas bajo riego, agotamiento de los acuíferos contiguos a las zonas urbanas, principios de desertificación, así como la eliminación de la cubierta vegetal nativa cuyas áreas han sido ocupados por el avance de la urbanización (Riemann y Ezcurra, 2007). Esto se traduce en inadecuadas políticas de usos asignadas a las unidades de gestión ambiental (UGAS), dando como resultado problemas de degradación, contaminación y agotamiento de los recursos que deberían ser el soporte de la sustentabilidad urbana.

En contraste los resultados que aquí se presentan para la ZMTPRT, pretenden dar una respuesta coherente y sustentable al crecimiento anárquico que se ha dado en esta región. No obstante que se utilizó parte de la misma información de cartografía del INEGI, como los modelos digitales de elevación (INEGI 1999a), las cartas topográficas y las cartas temáticas de aguas superficiales y subterráneas, también se utilizaron otras fuentes documentales que tiene cobertura estatal, como la cartografía geológica de Gastil et al. (1975), el Inventario Nacional Forestal (INE-SEMARNAP-I.G.UNAM, 1999), y la información generada sobre actividad tectónica y sismicidad (Cruz-Castillo, 2002). También se incorporó la información del Proyecto RADIOS (Rosquillas y Mendoza, 2001), permitiendo definir las zonas de sismicidad y tectónismo que presentan un alto riesgo, así como la presencia de fallas activas, información que en los documentos oficiales arriba citados no se contempla.

El resultado del manejo y análisis de las diferentes fuentes de información con el apoyo que ofrecen los SIGs y trabajo de campo, se obtuvo una zonificación para la ZMTPRT (SIDUE-SEDESOLUABC/ISS, 2005), totalmente diferente a la propuesta en el PEDUOT (SEDESOL-SIDUE-COLEF, 2003). (Fig. 3). En este sentido autores como Carsjens y Ligtenberg (2007) y Ojeda-Revah et al. (2008), utilizaron los SIG, como una herramienta poderosa en la planeación de los usos del suelo.

Por lo que respecta a la aplicación del modelo PER para la evaluación de la sustentabilidad en la ZMTPRT, generó resultados parciales, debido principalmente a que:

1. Las tres ciudades presentaban gran heterogeneidad en; población, dinámica económica, niveles de urbanización, industrialización, jerarquía urbana, densificación, capacidad de planeación y gestión en los ayuntamientos.

2. La información ambiental se encuentra dispersa en diferentes dependencias de los gobiernos municipal, estatal y federal.

3. En algunos casos se contó con información cualitativa de la operación de ciertos servicios.

4. No obstante que se tuvo accesos a los trabajos de Ordenamiento Territorial Estatal y Ecológico, pero debido a la escala y nivel de agregación de la información, no se pudo utilizar gran parte de esta, debido a su nivel de generalidad tanto de base de datos e información cartográfica.

5. Las tres ciudades no cuentan con el mismo nivel de información del listado.

6. Las estadísticas municipales adolecen de información ambiental periódica y sistemática, en algunos casos los estudios contienen información parcial o sectorial del comportamiento de algunas variables ambientales, o en el mejor de los casos es meramente cualitativa.

7. La accesibilidad a la información se encuentra limitada por parte de algunas dependencias gubernamentales.

8. En las diferentes dependencias de gobierno y sector privado no han entendido lo que implica el desarrollo sustentable, solamente lo utilizan como un discurso político, lo cual repercute en la administración y manejo de los recursos y servicios, de ahí que se dificulte su aplicación.

A pesar de estas limitaciones se pudo generar un diagnóstico, que permitió proponer algunas estrategias para minimizar o revertir problemas ambientales. Una de ellas radica en la necesidad de 
contar con una estructura de gestión general de la zona metropolitana y un grupo de trabajo que aborde en lo particular la temática ambiental y de sustentabilidad.

Repertti y Desthieux (2005), dicen que los desafíos del manejo urbano requieren de un manejo del territorio y una gran cantidad de información para poder establecer metas que conlleven a la sustentabilidad.

En este sentido se considera que la frontera norte de México, ha mostrado avances significativos en materia de sustentabilidad ambiental a través de un Programa Frontera XXI (1997) que empezó a operar desde 1983 desarrollado una serie de indicadores ambientales cuyo objetivo fue atacar los efectos a la salud causados por el deterioro del ambiente y a los ecosistemas.

Además en México se desarrolló un proyecto piloto para elaborar indicadores de sustentabilidad (INE-INEGI-SEMARNAP, 2000), tomando como base la propuesta de la OECD (1993), de sustentabilidad ambiental. A la fecha se tienen algunos avances en materia de planeación para el manejo sustentable del agua y de los residuos sólidos, asimismo se han probado algunas estrategias efectivas para abatir la contaminación del aire, a través de la instalación de estaciones de monitoreo, mediante el cual se han diseñado indicadores de sustentabilidad para mejorar la calidad del aire en toda la ZMTPRT (INE-SEMARNAP, DGE, 2000).

Rosquillas y Mendoza (2001), a través del PROYECTO RADIUS, abordan en forma muy precisa, la probabilidad de que se presente un evento sísmico de mediana a alta magnitud. En esta investigación sobre la sismicidad en la ZMTPRT, se hace referencia de los efectos que podría causar un sismo de mediana magnitud, cuales serían las instalaciones de mayor riesgo y cuales serían las acciones que antes, durante y después se tendrían que instrumentar para mitigar en lo posible los efectos devastadores de la actividad tectónica de toda la región. Si bien este reporte no hace referencia sobre indicadores, si permite generarlos, debido a la gran cantidad de información que aporta este documento.

Ojeda y Álvarez (2000), propone una estrategia para forestar las zonas susceptibles de deslizamientos de terreno, el propósito estabilizar y evitar en lo posible los movimientos de masa en las laderas. Estos mismos autores proponen crear un cinturón de áreas verdes que ayuden a mejorar la calidad ambiental de la ciudad de Tijuana y reducir su déficit.

Por otra parte se observa que entre los temas que requieren de mayor atención están; la necesidad de planear y gestionar los confinamientos para residuos peligrosos y no peligrosos, así como bases de datos que localicen las posibles zonas susceptibles de presentar riegos inducidos, al igual que la planeación y gestión para la protección de las escasas áreas verdes, de conservación ecológica a través de la conservación de la biodiversidad y de los diferentes hábitat que aún albergan especies de flora y fauna consideradas raras, amenazadas o en peligro de extinción (Wright y Vela, 2005).

Se asume que la planeación ambiental urbana de la ZMTPRT, requiere atender problemas de dispersión de los asentamientos humanos alentados por desarrollos industriales, que promueven el uso no sustentable del territorio, es decir examinar las posibilidades de densificación urbana, tomando en cuenta las restricciones físicas y biológicas que aporta la aptitud territorial, conjuntamente con incentivar e incrementar la movilidad de la población por medio de transporte público, actualizar y poner en práctica la regulación ambiental del parque vehicular privado con el propósito de disminuir y controlar los altos niveles de contaminación atmosférica e incentivar el cambio tecnológico del parque vehicular del transporte publico.

En lo referente a la propuesta de los modelos de ciclo cerrado de Tjallingii (1995), se debe de partir del principio de que la sustentabilidad se pierde cuando se empieza a extender mas allá de sus alrededores, por lo tanto van dependiendo más de corredores regionales a través de los cuales se canalizan los flujos de mercancías, recursos, energía, trabajo e información que necesitan para su sostenimiento dando como resultado que las megaciudades dependen más de regiones mucha más lejanas. Por lo tanto la zona de influencia de las megaciudades excede en mucho su territorio (Castaños, 2005). 
Analizadas estas variables, se considera que para poder proponer los modelos de ciclo y llevarlos a la práctica, es absolutamente indispensable contar con una amplia base de datos sobre los recursos disponibles en la región, aspecto que lo subsana la definición de la aptitud territorial.

Bajo esta premisa, la ordenación del territorio y definición de la actitud territorial y la utilización de los SIGs (Venegas, 2006), proporcionan información puntual y precisa de cual o cuales son los recursos naturales, renovables y no renovables de que se disponen, en que estado se encuentran y las amenazas por disminución o contaminación a los que están sujetos por la presión que se ejerce sobre ellos al hacer un uso no sustentable de los mismos.

Un ejemplo que ilustra esta aseveración; ¿cuales son los recursos hídricos que abastecen las zonas urbanas, donde están, cual o cuales son las amenazas a las que están sujetas, si son cuerpos de agua superficiales, presas o cuerpos de agua naturales o presas de almacenamiento, volúmenes aprovechables y si reciben descargas de la industria? (CNA, 2003).

En lo referente a los acuíferos, cual es el volumen que se debe de extraer, el cual debe estar acorde a los niveles de recarga, esto implica que se debe tener una amplia información de la precipitación media anual, ciclos recurrentes de precipitaciones excedentes o de sequías (Biondi et al., 2001; CNA, 2003), su nivel estático y dinámico, zonas e índices de recarga y si presentan algún tipo de contaminación, ya sea por uso intensivo de agroquímicos de la agricultura de riego, o por contaminación antropogénica, por industria o por verter las aguas municipales en las zonas de recarga, así como estrategias de su reutilización para evitar descargas a cuerpos de agua, ríos y finalmente al mar.

Una vez que se tiene esta información se puede construir un modelo de ciclo, como el que se presenta en la figura 4. Se considera que se tendría bastante certitud sobre la viabilidad y sustentabilidad del recurso agua, el cual es el más limitante en toda la región del noreste de México (CNA, 2003; Venegas, 2006).

\section{CONCLUSIONES}

El Ordenamiento Territorial es una valiosa herramienta para el manejo sustentable de los recursos, ofrece las bases científicas para la planeación ambiental de los usos del suelo, así como los criterios para su aprovechamiento bajo la óptica del desarrollo sustentable y una mejor evaluación y definición de las políticas asignadas a las unidades de gestión ambiental.

Asimismo la tendencia generalizada de la aplicación del ordenamiento territorial, en América Latina y Europa, ha incrementado su potencial con la utilización de los SIGs., ya que permiten la utilización de diferentes recursos digitales como; imágenes satelitales espectrales, fotografía área, modelos digitales de elevación y bases de datos lo que traduce en una definición puntual referente a la ubicación de los recursos existentes en el territorio, estableciendo las bases de la planeación ambiental y el manejo sustentable de los recursos que son la base económica de la sociedad.

En lo referente a los problemas que enfrenta el modelo PER para trabajar el diagnóstico a nivel urbano y/o regional, es debido a la carencia y deficiencia de información estadística ambiental y geográfica disponible en las dependencias de gobierno, debido a que a la fecha varias dependencias de los tres niveles de gobierno (Federal, Estatal y Municipal), no se han preocupado por elaborar y diseñar los formatos para la conformación de las bases de datos para crear sistemas de información geográficos que apoyen el seguimiento y evaluación del desempeño ambiental.

Esto se puede deber a la deficiencia o carencia de personal especializado en el área ambiental y la deficiencia con que operan los Departamentos de gestión ambiental del territorio, argumentando los administradores que es escaso el presupuesto que manejan y debido a esta situación, no es factible monitorear y mantener las bases de datos que se requieren para la gestión ambiental.

Respecto a la construcción de los modelos de ciclo para el manejo integral de los recursos en el territorio, permiten plantear y atacar el problema de la insustentabilidad en diferentes ámbitos de 
acción y escalas que van del nivel doméstico al nacional y acorde a su jerarquía y complejidad. El problema que se presenta para que estos modelos funcionen, es la deficiente información disponible sobre los recursos existentes en el territorio, para ello es absolutamente indispensable que se establezcan políticas y programas para la captura sistemática de este tipo de información, solamente así sería factible planear a largo plazo su aprovechamiento sobre la base de la sustentabilidad.

Por último, además del conocimiento puntual de los recursos existentes, sería deseable que cuando se haga la planeación regional y urbana, se considere el diseño de la infraestructura necesaria para establecer los modelos de ciclo cerrado, para todos y cada uno de los insumos que mantiene el metabolismo urbano, además de establecer políticas y normatividad para promover el reciclado y reutilización de los recursos ya utilizados, en lugar de desecharlos, verterlos a cuerpos de agua, o confinarlos en rellenos sanitarios, de esta forma, se tendría la posibilidad de prolongar su vida útil y mitigar problemas de contaminación ambiental como se propone en los modelos de ciclo cerrado.

\section{REFERENCIAS}

Binational Study: Migration between Mexico and the United States: Estudio binacional: migración entre México y los Estados Unidos. Acceso 4 de febrero 2008. (http://www.utexas.edu/lbj/uscir/ binational.html) (1997)

Biondi, F., A. Gershunov y D. R. Cayan; North Pacific Decadal Climate Variability since 1661, J. Climate: 14(1) 5-10 (2001)

Bustamante, J.A.; Migración irregular de México a Estados Unidos: 10 años de investigación del Proyecto Cañón Zapata. Frontera Norte: 12(23) 7-49 (2000)

Castaños, H.L.; Las megaciudades y la transición urbana. Revista Mexicana de Ciencias Políticas y Sociales, UNAM: XLVII (195) 95-121 (2005)

Carsjens, G.J. y A. Ligtenberg; A GIS-based support tool for sustainable spatial planning in metropolitan areas. Landscape Urban Plan.: 80 (1-2) 72-83 (2007)

CNA; Comisión Nacional del Agua. Programa Hidráulico de la Comisión nacional del Agua 20032006, Región I Península de Baja California. SEMARNAT-CNA-PND 194 pp (2003)

Cruz-Castillo, M.; Catálogo de las fallas regionales activas en el norte de Baja California, México. GEOS, Unión Geofísica Mexicana, 22 37-42 (2002)

CONEPO; Consejo Nacional de Población, Reporte sobre población en áreas urbanas. Poder Ejecutivo Baja California, 4 (2003)

Coubès, M.C.; Evolución del empleo fronterizo en los noventa: Efectos del TLECAN y de la devaluación sobre la estructura ocupacional. Frontera Norte: 15(30), 7-32 (2003)

DGE, Dirección General de Ecología; Plan de ordenamiento ecológico del Estado de Baja California. Periódico Oficial del Estado de B.C., Tomo CII (42) (1995)

Eastman, J.R.; IDRISI Kilimanjaro Version 14.002. Tutorial Clark Labs Clark University 950 Main Street Worcester, MA 01610-1477 USA (2003)

García, E.; Modificación al sistema de clasificación climatológica de Köppen (2 Ed.). UNAM, México DF, México pp. 252 (1981)

Gastil, R.G., R.P. Phillips y E.C. Allison; Reconnaissance geology of the State of Baja California. The Geological Society of America, Inc., Memoir: 140 (1975)

INEGI; Instituto Nacional de Estadística Geografía e Informática Modelos digitales de elevación Archivo I-11 Aguascalientes, México (1999a) 
INEGI; XII Instituto Nacional de Estadística Geografía e Informática, Censo industrial. Censos económicos. Aguascalientes, México (1999b)

INEGI; Instituto Nacional de Estadística Geografía e Informática, Anuario estadístico Baja California. Gobierno del Estado de Baja California (2006a) http://www.inegi.gob.mx/est/contenidos/espanol/ sistemas/conteo2005/localidad/iter/default.asp?s=est\&c=10395 Acceso 5 de agosto (2008)

INEGI; II Conteo de Población y Vivienda 2005. Disponible en línea: http://www.inegi.gob.mx/est/ contenidos/espanol/proyectos/conteos/conteo2005 (2006b)

INE-SEMARNAP-I.G.-UNAM. Inventario Nacional Forestal (1999)

INE-INEGI SEMARNAP; Sustainable Development Indicators of Mexico. INE-INEGI SEMARNAP. Mexico, D.F., 180, (2000)

INE-SEMARNAP, DGE; Programa Para Mejorar la Calidad del Aire de Tijuana-Rosarito 2000-2005 (2000)

INE-SEMARNAT; Manual del proceso de ordenamiento ecológico, México, D.F. (2006)

Kier, G., K. Muller y T. Rockwell; Origin of Regional Uplift Across Southern California and Northern Baja California. Eos, Trans. AGU Fall Meeting Suppl.: 82 (47), 1252 (2001)

Leitmann, J., (en línea) Rapid urban environmental assessment: lessons from cities in the developing world. Vol.2. Tools and Outputs. Washington, DC: The World Bank. 1-144 (1994) Acceso: 7 mayo (2008)

McGeough, U., D. Newman y J. Wrobel; Model for Sustainable Urban Design With Expanded Sections on Distributed Energy Resources. Sustainable Energy Planning Office. Gas Technology Institute and Oak Ridge National Laboratory (2004)

O'Connor, J. y C. Chase; Uplift of the Sierra San Pedro Mártir Baja California, Mexico. Tectonics: 8(4), 833-844 (1989)

OECD; Core set of indicators for environmental performance reviews. Environmental Monograph, 83, OECD, Paris (1993)

Ojeda, R.L. y G. Álvarez; La reforestación de Tijuana, Baja California como un mecanismo de reducción de riesgos naturales, Estudios Fronterizos: 1(2) 9-32 (2000)

Ojeda-Revah, L., G. Bocco, E. Ezcurra y I. Espejel: Land-cover/use transitions in the binational Tijuana River watershed during a period of rapid industrialization. Applied Vegetation Science: 11, 107-116 (2008)

Repertti, A. y G. Desthieux; A Relation Indicators Model for urban land-use planning and management: Methodological approach and application in two cases studies. Landscape Urban Plan.: 77 (1-2) 197-215 (2005)

Riemann, H. y E. Ezcurra; Endemic regions of the vascular flora of the peninsula of Baja California. J. Veg. Sci.: 18 (327-336) (2007)

Rosete, V.F.; Semblanza Histórico del Ordenamiento Ecológico Territorial en México INE-México, D. F. SEMARNAT (2006)

Rossiter, D. G., (en línea). Lecture Notes \& Reference: Methodology for Soil Resource Inventories . ITC Lecture Notes SOL.SRI. Enschede, the Netherlands: ITC. 132 pp. 2nd revised version, March2000. (First version February 1998) http://www.itc.nl/personal/rossiter/pubs/list.html Acceso: 5 mayo (2008) 
Rosquillas, N.A. y L. Mendoza; Proyecto RADIUS Caso Tijuana: Herramientas de evaluación de riesgo para diagnostico de áreas urbanas contra desastres sísmicos. H. XVI Ayuntamiento Constitucional de la ciudad de Tijuana, B.C. Dirección Municipal de Protección Civil- Centro de Investigación Científico y de Educación Superior de Ensenada División Ciencias de la Tierra, Departamento de Sismología. pp. 85 (2001)

Sanz, A.A. (en línea); La mayoría silenciada. Ciudades para un futuro más sostenible Boletín CF+S. Número 13. Julio 2000. http://habitat.aq.upm.es/boletin/n13/aasan.html Acceso: 2 de mayo 2008

Seattle/King County; Indicators of sustainable community. Sustainable Seattle, Washington, http://www.sustainableseattle.org, (1998) Acceso: 5 de mayo (2008)

SEDESOL., Secretaria de Desarrollo Social, Programa Nacional de Desarrollo Urbano y Ordenación

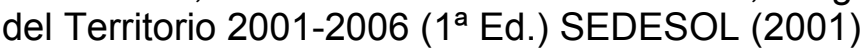

SEDESOL-SIDUE-COLEF; Programa estatal de desarrollo urbano y ordenamiento territorial. Documento de Trabajo, Mexicali, B.C. (2003)

SEMARNAT; Secretaria del Medio Ambiente y Recursos Naturales, Programa Nacional de Medio Ambiente y Recursos Naturales, 2001-2006, México D.F., México (2002)

SEMARNAP-SEDESOL-CONEPO-INEGI; Términos de Referencia Generales para la Elaboración del Programa Estatal de Ordenamiento Territorial, Versión Institucional (2000)

SIDUE-SEDESOL-UABC/ISS; Gestión Territorial de la Zona Metropolitana Tijuana-Tecate-Rosarito. Documento de trabajo, Instituto de Investigaciones Sociales, Universidad Autónoma de Baja California, Mexicali, B.C., México (2005)

Tjallingii, S.P.; Ecopolis, strategies for ecologically sound urban development. Backhuys, Leiden, pp 1-159 (1995)

United Nations, Spatial Planning Key Instrument for Development and Effective Governance with Special Reference to Countries in Transition UNITED NATIONS New York and Geneva (2008)

Venegas, C.F.R.; Aptitud territorial: una aproximación hacia la planeación del ordenamiento del territorio. UABC. Mexicali, B. C. México. 213 p. (2006)

Venegas, C.F. y R.I. Rojas; Instrumentos, Métodos y Técnicas Empleadas en la Planeación y Gestión del Territorio. In Planeación Urbana y Regional: Un enfoque hacia la sustentabilidad. R. I. Rojas C., pp. 235-295 Plaza y Valdés-UABC. México, D. F. (2006)

Wright, R. y R. Vela; Tijuana River Watershed Atlas San Diego State University, Institute for Regional Studies of the California (2005)

Zinck, J.A.; Physiography and Soils, Soil Survey Courses, International Institute for Aerospace and Earth Sciences, ITC. Enschede (1988) 
